\title{
Sozio-ethische Betrachtungen in Technikentwicklungsprojekten - Zeitverschwendung oder Erfolgsfaktor für Nutzerakzeptanz?
}

\author{
Stephanie Schmitt-Rüth • Martina Simon
}

Eingegangen: 18. Dezember 2019 / Angenommen: 7. März 2020 / Online publiziert: 17. März 2020

(C) Der/die Autor(en) 2020

Zusammenfassung Akzeptieren Menschen neue technologiebasierte Produkte oder Dienstleistungen nicht, so können diese aus technischer Perspektive noch so perfekt und nützlich sein - sie würden nicht in Anwendung und Nutzung kommen. Um mögliche Akzeptanzrisiken und Konfliktpotenziale in einem frühen Entwicklungsstadium zu identifizieren, ist es von entscheidender Bedeutung, sich einer sozioethischen Bewertung der Technik oder IT zu stellen. Auf diese Weise können Bedürfnisse, Interessen und Meinungen, insbesondere der von der Technik betroffenen Interessensgruppen, frühzeitig in den Entwicklungsprozess einbezogen werden. Im Rahmen dieses Beitrags wird ein generisches akzeptanzbasiertes Vorgehensmodell (HEART - Human-centered Evaluation of Acceptance and Risk Criteria for Technology) abgeleitet, um Fragestellungen der Mensch-Technik-Interaktion ergänzend zu Scrum oder Design Thinking-Methoden systematisch und effektiv erheben und bewerten zu können. Für die praktische Umsetzung von HEART wurde ein zweistufiges Verfahren gewählt, bestehend aus sogenannten Akzeptanz-Risiko-Workshops mit nachgelagertem Bewertungsfragebogen. Der Einbezug aller relevanten Stakeholder in Technikentwicklungsprojekte steht dabei im Mittelpunkt. Zwei Fallstudien in zwei Technikentwicklungsprojekten mit insgesamt 41 teilnehmenden Personen dokumentieren eine erste Anwendung von HEART in der Praxis und zeigen, dass sich das Konzept als geeignet erweist, um gemeinsam mit unterschiedlichen Zielgruppen deren Sichtweisen auf Technologie und ihre sozio-ethischen Risikofaktoren zu erheben und so integrative Forschung in Technikentwicklungsprojekten umzusetzen.

Aus Gründen der besseren Lesbarkeit wird im Rahmen dieses Beitrags das traditionelle generische Maskulinum verwendet. Es werden dabei immer alle Geschlechter im Sinne der Gleichbehandlung adressiert, die verkürzte Sprachform hat nur redaktionelle Gründe und beinhaltet keine Wertung.

S. Schmitt-Rüth $(\bowtie) \cdot$ M. Simon

Fraunhofer-Institut für Integrierte Schaltungen IIS, Nordostpark 84, 90411 Nürnberg, Deutschland E-Mail: stephanie.schmitt-rueth@iis.fraunhofer.de

M. Simon

E-Mail: martina.simon@iis.fraunhofer.de 
Schlüsselwörter Sozioethik · Technologieakzeptanz $\cdot$ Mensch-TechnikInteraktion · Nutzerintegration · Technikentwicklungsprojekte

\title{
Socio-ethical Considerations in Technology Development Projects-Waste of Time or Success Factor for User Acceptance?
}

\begin{abstract}
If people do not accept new technology-based products or services, from a technical perspective these can be as perfect and useful as they may be-they would not be applied and used. In order to identify possible acceptance risks and conflict potentials at an early stage of development, it is crucial to conduct a socioethical evaluation of the technology or IT. In this way, needs, interests and opinions, in particular of the stakeholders affected by the technology, can be included in the development process at an early stage. In the context of this contribution, a generic acceptance-based approach (HEART-Human-centered Evaluation of Acceptance and Risk Criteria for Technology) to be used in addition to Scrum and Design Thinking methods will be derived in order to systematically and effectively collect and evaluate questions of human-technology interaction. For the practical implementation of HEART, a two-stage procedure was chosen, consisting of socalled acceptance-risk workshops with a subsequent evaluation questionnaire. The focus here is on the inclusion of all relevant stakeholders in technology development projects. Two case studies in two technology development projects with 41 participants document a first practical application of HEART and demonstrate that HEART is suitable to collect and discuss views on technology and its socio-ethical risk factors together with various stakeholder groups and thus helps to implement integrative research in technology development projects.
\end{abstract}

Keywords Socio-ethical · Technology acceptance $\cdot$ Human-Technology Interaction · Stakeholder Integration · Technology R\&D Projects

\section{Einleitung}

„Technology is neither good nor bad; nor is it neutral.“ - 1986 beschrieb Kranzberg (1986) in seinem ersten Technologiegesetz das Faktum, dass Technologie in ihrer (Aus-)Wirkung keineswegs immer eindeutig wahrgenommen wird, sondern stets in Abhängigkeit von Kontext und individueller Perspektive unterschiedlich beurteilt werden kann. Der Einbezug dieser unterschiedlichen Wahrnehmungen und Perspektiven, insbesondere zu Beginn von Technikentwicklungsprojekten, stößt jedoch bei Entwicklern nicht immer auf Gegenliebe: zu groß ist die Angst, dass der Einbezug unterschiedlicher Meinungen, insbesondere potentieller zukünftiger Nutzer, auf modifizierungsbedürftige Aspekte hinweist, welche bereits geplante Entwicklungsschritte vereiteln könnten.

Dabei ist es gerade diese Phase, in der Änderungen vorgenommen werden könnten, bevor sie in späteren Phasen der Entwicklung unangenehm und kostspielig werden würden (Palm und Hansson 2018). Neuartige Technik wirft eine Reihe sozial, ethisch und rechtlicher Fragestellungen auf, wie Selbst- und Fremdbestimmung 
oder Teilhabe durch Technik. Die Identifizierung dieser Fragestellungen zur weiteren Berücksichtigung und Integration in ein Technikentwicklungsprojekt ist dabei nicht immer leicht (Carsten Stahl 2011).

Um dieser Problematik zu begegnen, wird ein Vorgehensmodell entwickelt, das ergänzend zu Scrum oder Design Thinking-Methoden eingesetzt werden kann und soziale, ethische und rechtliche Aspekte unter Einbezug potenzieller Nutzergruppen identifizieren soll. Durch eine Bewertung so aufgedeckter Chancen, Probleme und potenzieller Konfliktfelder sowie eine Identifikation zukünftiger Handlungsfelder soll Akzeptanzproblemen seitens der Nutzer frühzeitig begegnet werden.

Im Folgenden wird zunächst der theoretische Hintergrund des Vorgehensmodells erläutert. Im Anschluss werden die Bestandteile des Modells aufgezeigt und dessen praktische Umsetzbarkeit im Rahmen einer Case Study diskutiert.

\section{Ableitung eines akzeptanzbasierten Frameworks für sozio-ethische Betrachtungen in Technikentwicklungsprojekten}

\subsection{Theoretischer Hintergrund}

Eines der im Rahmen von technikbasierten Forschungsprojekten derzeit am meisten eingesetzten ethischen Frameworks im deutschsprachigen Raum ist das sogenannte MEESTAR-Modell (Modell zur ethischen Evaluierung sozio-technischer Arrangements; (Manzeschke 2015)). Das Modell beschreibt ,,soziale und technische Voraussetzungen, die zur Entwicklung und dem möglichen Einsatz dieser Systeme führt" (Manzeschke 2015). Es besteht insgesamt aus sieben ethischen Bewertungsdimensionen (Fürsorge, Selbstbestimmung, Sicherheit, Gerechtigkeit, Privatheit, Teilhabe und Selbstverständnis), die auf drei Ebenen (Individuum, Organisation, Gesellschaft) betrachtet werden. MEESTAR wurde aus dem Gesundheitsbereich heraus entwickelt und erfährt stetige Weiterentwicklungen (z. B. Weber 2016). Weber (2016) verweist in seinem Beitrag darauf, dass zwischen Technikgestaltung und Technikakzeptanz eine enge Verbindung besteht: Akzeptanz gilt nämlich als dominanter Erfolgsfaktor für neue technikbasierte Lösungen (Königstorfer 2008).

Unter Akzeptanz versteht man dabei „die positive Annahmeentscheidung einer Innovation durch die Anwender" (Simon 2001), wobei meist zwischen Einstellungsund Verhaltensakzeptanz unterschieden wird (Kollmann 1998). So kann zwar aus sozio-ethischer Perspektive ein System/eine Technik als „korrekt“ bewertet werden, muss deshalb aber noch lange nicht unter den Stakeholdern und potentiellen Nutzern akzeptiert werden. Genauso ist der Umkehrschluss möglich: ethisch verwerfliche Systeme werden ohne Bedenken von den Zielgruppen akzeptiert. Aus Sicht der Akzeptanzforschung beziehen sich Akzeptanzfaktoren auf das Zusammenwirken von Akzeptanzsubjekt, Akzeptanzobjekt und Akzeptanzkontext (Lucke 1995). Das Akzeptanzsubjekt entwickelt so eine Einstellung gegenüber einem Akzeptanzobjekt, was wiederum zu einer Handlung führt. Bei dem Akzeptanzobjekt handelt es sich um das Angebotene, Vorhandene oder Vorgeschlagene. Der Akzeptanzkontext bezieht sich auf alle Faktoren, die nicht im Akzeptanzsubjekt und -objekt miteinbegriffen sind und auf die Nutzungssituation einwirken. 


\subsection{Vorgehen und Ergebnis}

Vor dem Hintergrund eines potentiellen Zusammenwirkens von Akzeptanz und sozio-ethischen Aspekten ist es von Bedeutung zu verstehen, welche generischen Begrifflichkeiten bei der Betrachtung von Technik im Allgemeinen verwendet werden. Hierzu wurde eine explorative Literaturanalyse durchgeführt, die folgende vier am häufigsten adressierten sozio-ethischen Themenbereiche identifizierte:

- Sicherheit. Das Grundbedürfnis nach Sicherheit beschreibt zum einen die Unversehrtheit der Gesundheit, die sich aus dem körperlichen und psychischen Wohlbefinden zusammensetzt. Hinzu kommen weitere Aspekte wie Schädigungsfreiheit, Privatsphäre sowie Vertraulichkeit und Datenschutz. Schädigungsfreiheit stellt auf Aspekte wie Zuverlässigkeit und Fahrlässigkeit ab und adressiert die Frage: Welche Gefahren für das Wohl der Stakeholder sind mit dem Einsatz des Systems verbunden?

- Gerechtigkeit \& Teilhabe. Begrifflichkeiten wie Solidarität, Fairness, Gleichheit/ Ungleichheit, Diskriminierung, Stigmatisierung, Rechte, Inklusion \& Exklusion, Erhältlichkeit, Bezahlbarkeit, Besitz, universeller Zugang, Beschäftigung/Arbeit formen diese Oberkategorie. Wird der Platz der Nutzenden in der Gesellschaft beeinflusst? Wie wirkt sich das System auf den gesellschaftlichen Zusammenhalt aus? Auch Chancengleichheit wird adressiert: Ergeben sich für alle Nutzergruppen dieselben Chancen und Möglichkeiten? Sind bestimmte Gruppen von der Nutzung des Systems ausgeschlossen? Entstehen bestimmten Nutzergruppen besondere Vorteile/Nachteile durch das System? Beeinträchtigt das System die Teilhabe bestimmter Gruppen? Diverse Menschenbilder/Normierung: Wie wird mit Unterschieden umgegangen? Werden diese anerkannt? Stigmatisierung: Können Anwender durch das System bloßgestellt werden? Diskriminierung: Gibt es Merkmale die manche Anwendergruppen benachteiligen? Zuletzt ist Wertschätzung zu nennen: Werden die Nutzenden wertschätzend behandelt?

- Nachhaltigkeit. Aspekte wie Effizienz, Wirksamkeit, soziale Nachhaltigkeit, ökonomische Nachhaltigkeit, Umwelt, Rentabilität, Kosten determinieren die dritte Kategorie. Wie wirkt sich die Innovation/das technische System auf den Erhalt der Lebenswelt der Nutzenden aus? Hierbei sind Einflüsse auf das Wirtschaftsbzw. Versorgungssystem sowie die ökologische Umwelt zu thematisieren.

- Selbstbestimmung. Dieser Begriff umfasst Aspekte wie Abhängigkeit/Unabhängigkeit, Kontrollierbarkeit, Aufklärung und Anwendungsfreundlichkeit. Sind die Nutzenden frei von jeglicher Fremdbestimmung und können sie über die Nutzung des Systems selbst entscheiden? Ist die Wahlfreiheit der Nutzenden gewährleistet? Vertraulichkeit, Privatheit und Datenschutz adressieren die Relevanz und Sensibilität von Daten: Ist der Schutz von Daten und Inhalten gewährleistet? Ist der Zugriff auf Daten und Inhalte klar geregelt? Werden durch das System personenbezogene Daten erfasst? Ist eine Erfassung erforderlich? Ist die Erfassung kritisch zu sehen? Können die Daten missbraucht werden? Können Daten oder Inhalte wieder gelöscht werden? Können die Inhalte oder erhobenen Daten vom Nutzer eingesehen werden? Erhält der Nutzer Einsicht, wer Zugriff auf Inhalte oder erhobene Daten hat? 


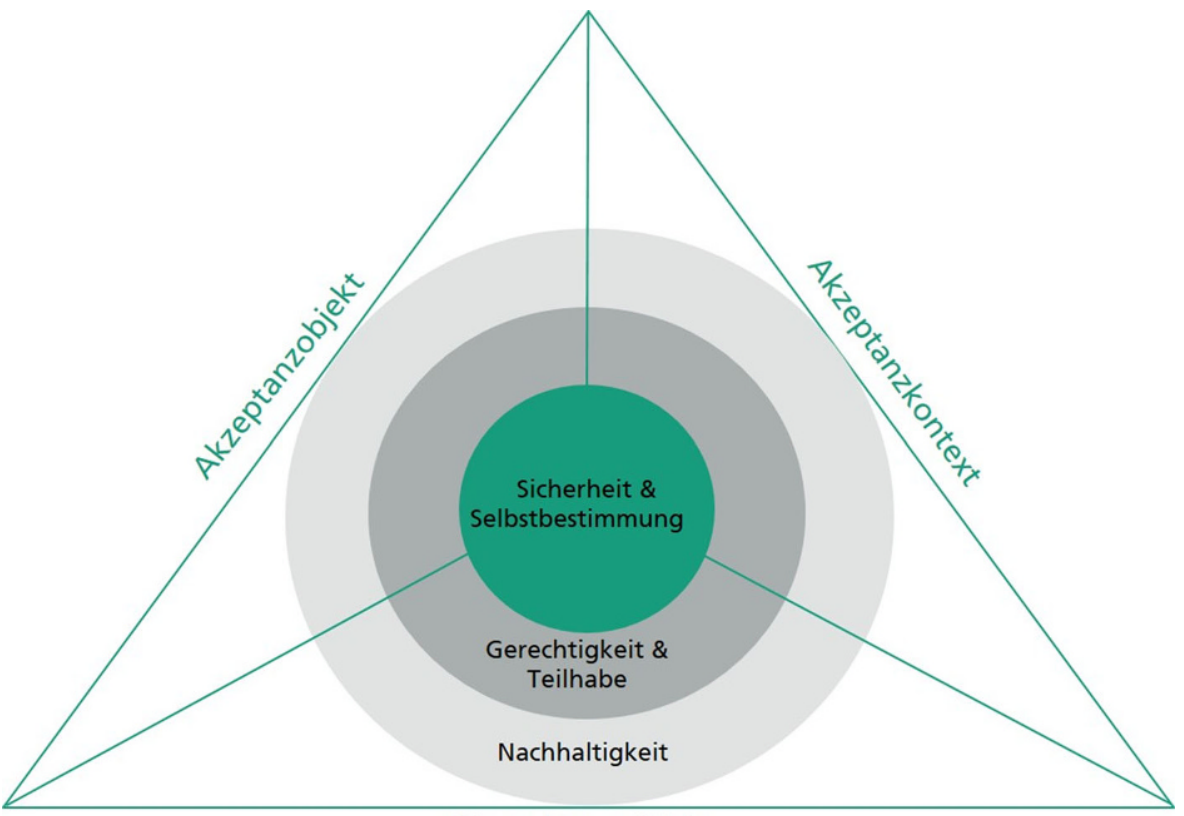

Akzeptanzsubjekt

Abb. 1 Akzeptanzbasiertes Framework zur sozio-ethischen Betrachtung

Es wird deutlich, dass die vier gebildeten generischen Oberkategorien in Tradition mit existierenden ethischen Modellen stehen und ebenfalls den drei Betrachtungsebenen des MEESTAR-Modells (Manzeschke 2015) zugeordnet werden können:

- Ebene individueller Bedürfnisse: Sicherheit (Schädigungsfreiheit; Verantwortung) und Selbstbestimmung (Wahlfreiheit und Autonomie; Vertraulichkeit und Privatheit)

- Position des Individuums in der Gesellschaft: Gerechtigkeit \& Teilhabe (Chancengleichheit; Diskriminierung und Stigmatisierung)

- Überindividuelle, gesamtgesellschaftliche Themen: Nachhaltigkeit (Gesundheitsund Wirtschaftssystem; Gesellschaft und Umwelt)

Diese Ebenen können nun in Abb. 1 als konzentrische Kreise dargestellt werden. In Kombination mit den Akzeptanzkomponenten, welche als tragende Seiten eines gleichseitigen Dreiecks auf die Kreise wirken, bilden sie so das akzeptanzbasierte Framework sozio-ethischer Betrachtungen.

\section{HEART - Entwicklung eines praxisorientierten Vorgehensmodells zur akzeptanzbasierten Bewertung sozio-ethischer Risikoaspekte in Technikentwicklungsprojekten}

Um das im vorherigen Kapitel vorgestellte akzeptanzbasierte Framework für einen Einsatz in der Praxis nutzbar zu machen, muss ein umsetzbares Vorgehensmodell 
entwickelt werden, in dem Chancen, Probleme und potentielle Konfliktfelder sowie zukünftige Handlungsfelder, die mit der zu entwickelnden Technik verbunden sind, gemeinsam mit den Nutzern identifiziert, bewertet und für die weitere Entwicklung berücksichtigt werden können.

Für die praktische Umsetzung des Frameworks in ein Vorgehensmodell wurde auf Basis der Anforderungen - ein zweistufiges Verfahren gewählt. Das Vorgehensmodell, das im nachfolgenden HEART (Human-centered Evaluation of Acceptance and Risk Criteria for Technology) benannt wird, besteht aus einem „Akzeptanz-Risiko-Workshop“" und einem nachgelagerten Bewertungsfragebogen (siehe Abb. 2).

Den ersten Bestandteil des Verfahrens bildet der sog. Akzeptanz-Risiko-Workshop, dessen Ziel es ist, mit Vertretern verschiedener Nutzergruppen in Gruppenarbeit eine Sammlung kritischer Aspekte zu den identifizierten sozio-ethischen Themenbereichen herauszuarbeiten, die es zu berücksichtigen gilt, um Akzeptanzprobleme seitens der Nutzer in Technikentwicklungsprojekten zu vermeiden. Die Identifikation der in den Akzeptanz-Risiko-Workshop zu involvierenden Nutzervertreter erfolgt dabei unter Rückgriff auf Stakeholderanalysen aus der Phase der Initialisierung des jeweiligen Entwicklungsprojekts. Diese Nutzervertreter diskutieren im Akzeptanz-Risiko-Workshop unter Anwendung der Methode der Fokusgruppe nach einer allgemeinen Vorstellung des Technikentwicklungsprojekts in Kleingruppen die vier Themenbereiche Sicherheit, Selbstbestimmung, Gerechtigkeit \& Teilhabe sowie Nachhaltigkeit mit ihren jeweiligen Unterkategorien. Den einzelnen Themenbereichen samt Unterkategorien wird dabei in der praktischen Umsetzung im Idealfall ein eigener Tisch gewidmet, wobei die Unterkategorien jeweils in Form von großen Rädern auf den zugehörigen Thementischen liegen (vgl. hierzu Abb. 2). Die einzelnen Themenbereiche werden von den Kleingruppen jeweils nacheinander durchlaufen, wobei jeder Themenbereich bzw. Tisch von einem Moderator begleitet wird, der inhaltlich in das jeweilige Thema mit seinen Unterkategorien einführt und die von den Teilnehmern in der gemeinsamen Diskussion hervorgebrachten kritischen Aspekte sowie ggf. Lösungsvorschläge auf Post-its notiert und diese auf den UnterkategorieRädern anbringt. Zusätzlich zu diesen Notizen wird der gesamte Akzeptanz-RisikoWorkshop mit Einverständnis der Teilnehmer auf Tonband aufgezeichnet. Sobald alle Gruppen alle sozio-ethischen Themenbereiche bearbeitet haben, werden die Ergebnisse gemeinsam betrachtet und erste kritische bzw. weniger kritische Aspekte definiert.

Nun genügt die einfache Sammlung der relevanten Aspekte per se noch nicht, um spezifische nachgelagerte Entwicklungstätigkeiten/-anpassungen vornehmen zu können. Eine Priorisierung der Bewertungsdimensionen wird oftmals gefordert (vgl. z.B. Weber 2016). Der zweite Bestandteil des HEART-Ansatzes verfolgt somit das Ziel, die im Rahmen des Akzeptanz-Risiko-Workshops identifizierten Aspekte mittels eines nutzerzentrierten quantitativen Bewertungssystems für Technikentwicklungsprojekte nutzbar zu machen und geht somit über die erste, qualitative Identifikation und Bewertung der Aspekte im Workshop hinaus. Hierzu werden die im Akzeptanz-Risiko-Workshop identifizierten Aspekte im Rahmen der Auswertung aggregiert und anschließend in einen Bewertungsfragebogen überführt, der den Workshop-Teilnehmern nachgelagert zur Beantwortung vorgelegt wird. Im Bewertungsfragebogen wird eine 5-stufige Likert-Skala mit den Endpunkten 1 (=Aspekt 


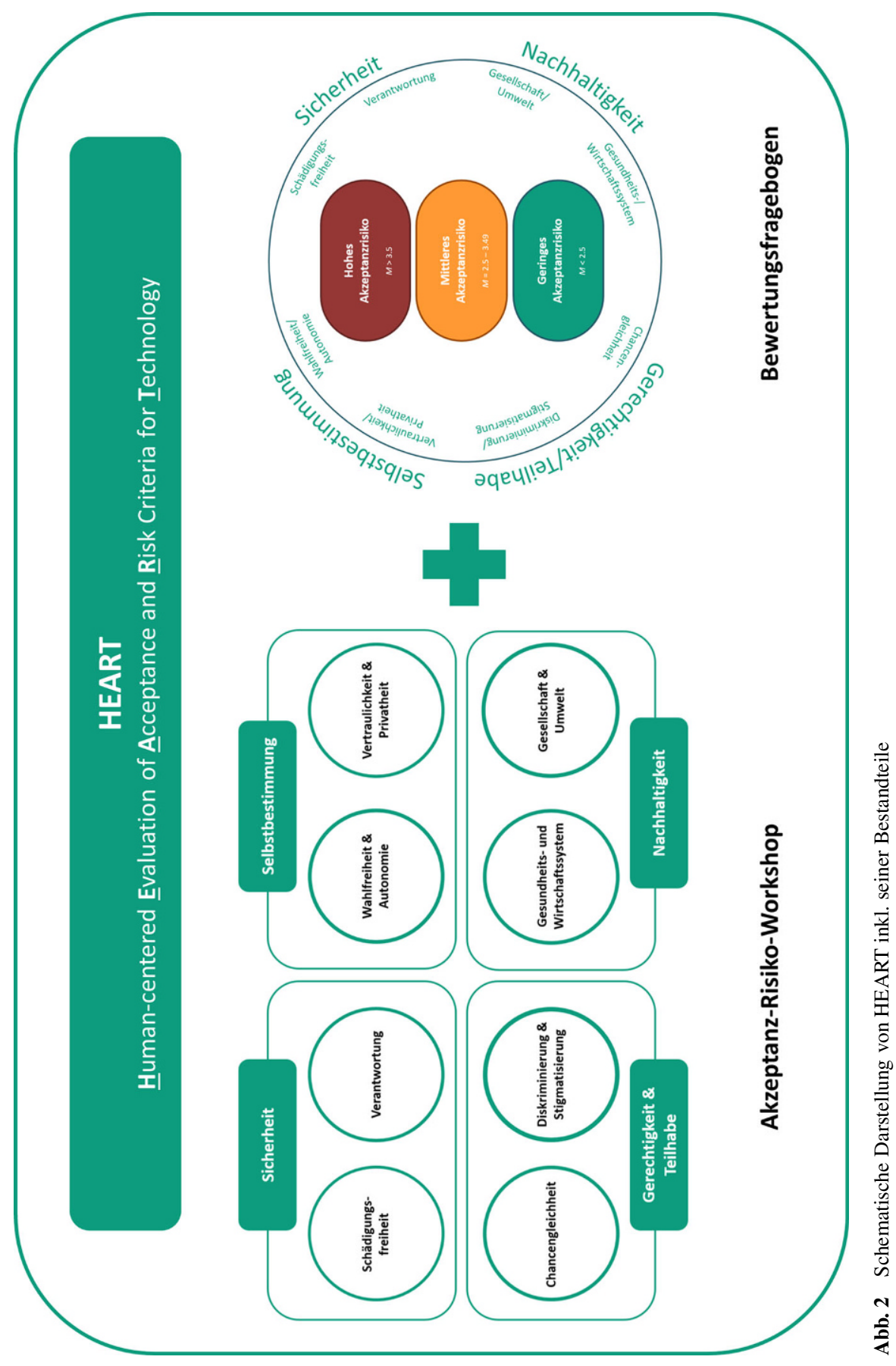


stellt ein sehr geringes Akzeptanzrisiko dar) und 5 (=Aspekt stellt ein sehr hohes Akzeptanzrisiko dar) eingesetzt. Im Rahmen von deskriptiven Analysen können so Aspekte identifiziert werden, die aggregiert über alle teilnehmenden Nutzervertreter ein hohes Akzeptanzrisiko darstellen $(M>3,5)$. Ebenso können Aspekte mit mittlerem $(M=2,5-3,49)$ und geringem $(M<2,5)$ Akzeptanzrisiko identifiziert werden. Die Ergebnisse können so in Form einer tabellarischen und nach Grad des Akzeptanzrisikos gegliederten Ergebnisdokumentation direkt an verschiedene Stakeholder innerhalb des Projekts weitergegeben werden und dort als Priorisierungsgrundlage für weitere Entwicklungs- und Evaluationsschritte dienen.

\section{Fallbeispiel aus der Praxis - Wenn HEART auf reale Technikentwicklungsprojekte trifft}

Der HEART-Ansatz wurde in zwei geförderten Technikentwicklungsprojekten, „ERIK“1 und „DIGI-ORT“2, praktisch angewendet und konnte dort in vier Akzeptanz-Risiko-Workshops mit insgesamt 41 Teilnehmern hinsichtlich seiner Praxistauglichkeit, Effektivität und Effizienz überprüft werden. Anhand des Projekts ERIK werden nachfolgend beispielhaft Umsetzung und Ergebnisse des HEARTAnsatzes dargestellt.

\subsection{Case Study: ERIK}

Im vom Bundesministerium für Forschung und Entwicklung geförderten Forschungsprojekt ERIK wird eine Roboterplattform zur Unterstützung neuer Interaktionsstrategien in der Therapie von Kindern mit eingeschränkten sozio-emotionalen Fähigkeiten entwickelt. Diese Roboterplattform in Gestalt des humanoiden Roboters Pepper ${ }^{3}$ soll auf Basis von Mimik, Sprache und Puls die Emotionen eines autistischen Kindes erkennen und ein Echtzeitfeedback generieren. Das Kind kann so durch das Robotersystem lernen, die Emotionen seines Gegenübers selbst richtig zu erkennen und darzustellen, wodurch die sozio-emotionalen Kompetenzen des Kindes gesteigert werden können (Simon et al. 2019).

Eine Herausforderung des Projekts liegt unter anderem in der Zielgruppe, die der Gruppe der sogenannten „Vulnerable Consumers“ (Baker et al. 2005) zugeordnet werden kann: Menschen bzw. im engeren Sinne Kinder im Autismus-Spektrum. In Bezug auf die praktische Umsetzung des HEART-Ansatzes galt es daher, neben der Prüfung der allgemeinen Praxistauglichkeit insbesondere auch festzustellen, ob das Konzept für die Gruppe der „Vulnerable Consumers“ geeignet ist und angewendet werden kann.

\footnotetext{
1 https://www.technik-zum-menschen-bringen.de/projekte/erik

2 https://digitales-dorf.bayern/index.php/die-modelldoerfer/digitales-gesundheitsdorf-oberes-rodachtal/

3 https://www.softbankrobotics.com/corp/robots/
} 


\subsection{Praktische Umsetzung und Ergebnisse des Akzeptanz-Risiko-Workshops}

Im Projekt ERIK wurden zwei separate Akzeptanz-Risiko-Workshops mit unterschiedlichen Nutzervertretergruppen durchgeführt, die im Januar 2019 in Nürnberg bzw. Berlin stattfanden. Insgesamt nahmen 25 Personen teil, darunter Projektbeteiligte, Erwachsene im Autismus-Spektrum, Eltern/Angehörige, Therapeuten sowie Wissenschaftler mit Forschungsschwerpunkt Ethik. Die beiden Workshops wurden von jeweils zwei Moderatoren und einer Hilfskraft begleitet und zur besseren Auswertbarkeit auf Tonband aufgezeichnet. Die Durchführung erstreckte sich auf einen zeitlichen Rahmen von 4h inklusive Pausen, wobei beide Akzeptanz-RisikoWorkshops sowohl hinsichtlich ihres zeitlichen als auch ihres inhaltlichen Ablaufs identisch waren: Nach einer jeweils rund 45-minütigen Vorstellung des Forschungsvorhabens wurden in anschließender Gruppenarbeit mit zwei Gruppen im Wechsel die sozio-ethischen Themenbereiche Sicherheit, Selbstbestimmung sowie Nachhaltigkeit und Gerechtigkeit und Teilhabe diskutiert. Pro Themenbereich waren hierfür 30 min vorgesehen, wobei die Unterkategorien pro Themenbereich (z. B. Schädigungsfreiheit und Verantwortung im Themenbereich Sicherheit; vgl. Abb. 2) jeweils nacheinander diskutiert wurden. Sowohl als kritisch erachtete Aspekte als auch Lösungsvorschläge seitens der Workshop-Teilnehmer wurden von den Moderatoren auf Post-its notiert und den jeweiligen Unterkategorien zugeordnet. Nach der Gruppenarbeitsphase, die von zwei jeweils 15-minütigen Pausen begleitet war, wurden die erarbeiteten Punkte gemeinsam betrachtet und mögliche Lösungsvorschläge diskutiert (siehe Abb. 3). Im Rahmen dieser rund 45-minütigen Abschlussphase wurde auch versucht, mittels einer Bepunktung der gesammelten Post-its bereits im Vorfeld besonders kritische Aspekte zu identifizieren, was jedoch aufgrund der Vielzahl der

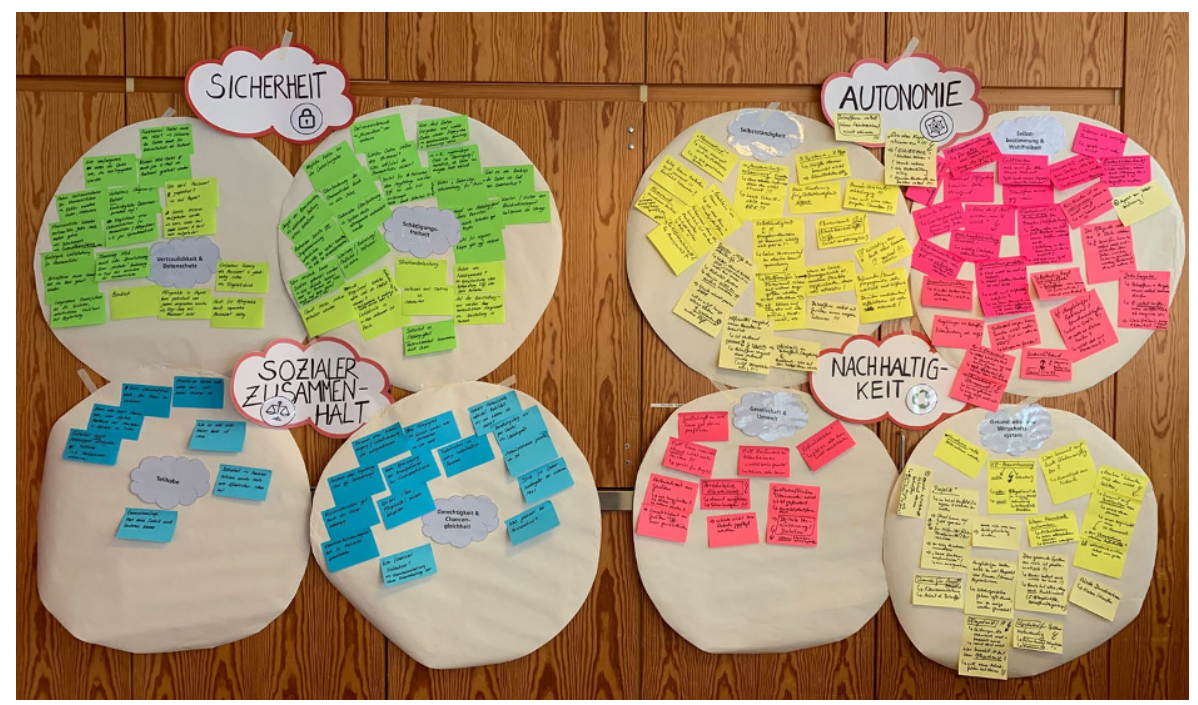

Abb. 3 Beispielhafte Darstellung einer Ergebnispräsentation im Rahmen der gemeinsamen Abschlussdiskussion 
Post-its, auf denen Aspekte bedingt durch die Gruppenarbeit teilweise auch doppelt genannt waren, nicht gelang.

Für die Auswertung der Akzeptanz-Risiko-Workshops wurde sowohl auf die Post-it-Sammlungen zu den einzelnen Themenbereichen als auch auf die Tonbandaufzeichnungen zurückgegriffen, die mittels MAXQDA in Anlehnung an Mayring (2002) transkribiert und ausgewertet wurden. In den beiden Workshops wurden insgesamt 307 Post-it-Notizen sowie 15h Tonmaterial generiert (Simon et al. 2019). Nach Abgleich der Post-it-Notizen mit den transkribierten Texten wurden aus diesen relevante Einzelaussagen codiert: So konnten 708 Einzelaussagen erstellt werden, die innerhalb ihrer jeweiligen Themenfelder zu Handlungsfeldern, d.h. potenziell kritischen Aspekten, aggregiert wurden und die Basis für die Bewertungsfragebögen bildeten.

\subsection{Praktische Umsetzung und Ergebnisse des Bewertungsfragebogens}

Basierend auf den Ergebnissen der Akzeptanz-Risiko-Workshops wurden mittels LimeSurvey Online-Fragebögen zu 55 Handlungsfeldern bzw. potenziell kritischen Aspekten in ERIK erstellt. Diese potenziell kritischen Aspekte wurden den Teilnehmern der beiden Akzeptanz-Risiko-Workshops zur Bewertung auf 5-stufigen-LikertSkalen vorgelegt ( $1=$ Aspekt stellt ein sehr geringes Akzeptanzrisiko dar; 5= Aspekt stellt ein sehr hohes Akzeptanzrisiko dar). Der Zugang zu den Online-Fragebögen erfolgte dabei mittels eines generischen Links, der den Workshop-Teilnehmern jeweils in einer E-Mail mitgeteilt worden war.

Die Online-Umfrage in ERIK wurde vom 15.03.-25.03.2019 durchgeführt und von 14 Personen beantwortet. Die 55 Handlungsfelder bzw. potenziell kritischen Aspekte wurden im Anschluss an die Online-Umfrage deskriptiv mittels SPSS ausgewertet, wobei in Aspekte unterschieden wurde, die ein hohes $(M>3,5)$, mittleres $(M=2,5-3,49)$ sowie ein niedriges Akzeptanzrisiko $(M<2,5)$ aufweisen. Die identifizierten kritischen Aspekte wurden in Anlehnung an Lucke (1995) zusätzlich explizit hinsichtlich des Ursprungs des Akzeptanzrisikos in Aspekte, deren Akzeptanzrisiko a) vom Akzeptanzsubjekt, b) vom Akzeptanzobjekt bzw. c) vom Akzeptanzkontext abhängt, gegliedert (Simon et al. 2019).

Insgesamt konnten 5 Aspekte mit hohem Akzeptanzrisiko, 35 Aspekte mit mittlerem sowie 15 Aspekte mit geringem Akzeptanzrisiko identifiziert werden (Simon et al. 2019). Tab. 1 zeigt beispielhaft die Aspekte mit hohem Akzeptanzrisiko in ERIK.

Mit Hinblick auf die geringe Teilnehmerzahl von $N=14$ als auch die teilweise hohen Standardabweichungen, die sich in allen drei Akzeptanzbereichen finden, sind neben den Aspekten mit hohen Akzeptanzrisiko auch die Aspekte mit mittlerem und niedrigem Akzeptanzrisiko in die weitere Entwicklung einzubeziehen (Simon et al. 2019). Hierzu wurde eine tabellarische Dokumentation inkl. Risikoeinschätzung erstellt, die den technischen Entwicklern zur Verfügung gestellt wurde und im weiteren Projektverlauf mit den Nutzern von ERIK hinsichtlich ihrer Umsetzung evaluiert werden soll. 
Tab. 1 Aspekte mit hohem Akzeptanzrisiko in ERIK als Ergebnis aus den Online-Bewertungsfragebögen (Simon et al. 2019)

\begin{tabular}{|c|c|c|c|c|}
\hline \multicolumn{5}{|l|}{ ERIK } \\
\hline Handlungsfeld & Themenbereich & $\begin{array}{l}\text { Ursprung Ak- } \\
\text { zeptanzrisiko }\end{array}$ & $\begin{array}{l}\text { Einschätzung } \\
\text { Risiko }\end{array}$ & $N$ \\
\hline Datenzugriff durch Unbefugte & Selbstbestimmung & Akzeptanzobjekt & $\begin{array}{l}M=4,07 \\
S D=1,07\end{array}$ & 14 \\
\hline $\begin{array}{l}\text { Erlernen von roboterhaftem/sozial } \\
\text { künstlichem Verhalten/künstlichen } \\
\text { Emotionen }\end{array}$ & $\begin{array}{l}\text { Gerechtigkeit } \\
\& \text { Teilhabe }\end{array}$ & Akzeptanzobjekt & $\begin{array}{l}M=3,86 \\
S D=1,01\end{array}$ & 14 \\
\hline $\begin{array}{l}\text { Rückmeldung falscher Emotionen } \\
\text { von Pepper an Kinder (Eskalation des } \\
\text { Spiels) }\end{array}$ & Sicherheit & Akzeptanzobjekt & $\begin{array}{l}M=3,64 \\
S D=1,45\end{array}$ & 14 \\
\hline $\begin{array}{l}\text { Normierung von Menschen im autis- } \\
\text { tischen Spektrum }\end{array}$ & $\begin{array}{l}\text { Gerechtigkeit } \\
\& \text { Teilhabe }\end{array}$ & Akzeptanzobjekt & $\begin{array}{l}M=3,60 \\
S D=1,11\end{array}$ & 13 \\
\hline $\begin{array}{l}\text { Missbrauch/Manipulierbarkeit der } \\
\text { Daten und Software durch Dritte }\end{array}$ & Selbstbestimmung & Akzeptanzobjekt & $\begin{array}{l}M=3,54 \\
S D=1,27\end{array}$ & 13 \\
\hline
\end{tabular}

\section{Diskussion und weiterer Forschungsbedarf}

Sowohl im Rahmen der dargestellten Case Study ERIK als auch im zweiten Projekt DIGI-ORT konnte der HEART-Ansatz mit verschiedenen Nutzergruppen, u. a. auch Vertretern der Gruppe der „Vulnerable Consumers“ (Baker et al. 2005), hinsichtlich seiner Praxistauglichkeit, Effektivität und Effizienz überprüft und daraus Stärken und Schwächen identifiziert werden.

\subsection{Stärken des HEART-Ansatzes}

Als eine Stärke des HEART-Ansatzes hat sich dessen Flexibilität, insbesondere bezüglich der Gestaltung und Durchführung des Akzeptanz-Risiko-Workshops, erwiesen: Je nach zeitlichen Ressourcen der Teilnehmer können die einzelnen sozioethischen Themenbereiche inkl. vorheriger Einführung in das Technikentwicklungsprojekt in einem Zeitrahmen von 2,5 bis $4 \mathrm{~h}$ diskutiert werden, wobei die einzelnen Bestandteile des Akzeptanz-Risiko-Workshops sowohl hinsichtlich ihrer Dauer als auch ihres Ablaufs flexibel angepasst werden können. Abb. 4 zeigt diese Variationsmöglichkeiten der Akzeptanz-Risiko-Workshops in einem Vergleich der beiden Entwicklungsprojekten ERIK und DIGI-ORT.

Wie in Abb. 4 zu sehen, kann beispielsweise die Dauer der Vorstellung des Forschungsvorhabens zu Beginn des Workshops variiert werden. Die Erfahrung hat gezeigt, dass es günstig ist, der Vorstellung des Forschungsvorhabens zu Beginn des Workshops mehr Zeit einzuräumen, da hier bereits zahlreiche Fragen und erste Diskussionspunkte seitens der Workshopteilnehmer aufkommen, die bei einem zu knappen Zeitrahmen nicht ausreichend behandelt werden können. Ein zeitlicher Mehraufwand kann sowohl durch eine zeitliche Kürzung der Abschlussdiskussion als auch durch eine zeitliche Kürzung aller oder einzelner Gruppenarbeitsphasen bzw. Verzicht auf die Gruppenarbeitsphasen kompensiert werden. Letzteres bietet sich insbesondere dann an, wenn der Akzeptanz-Risiko-Workshop aus weniger als 


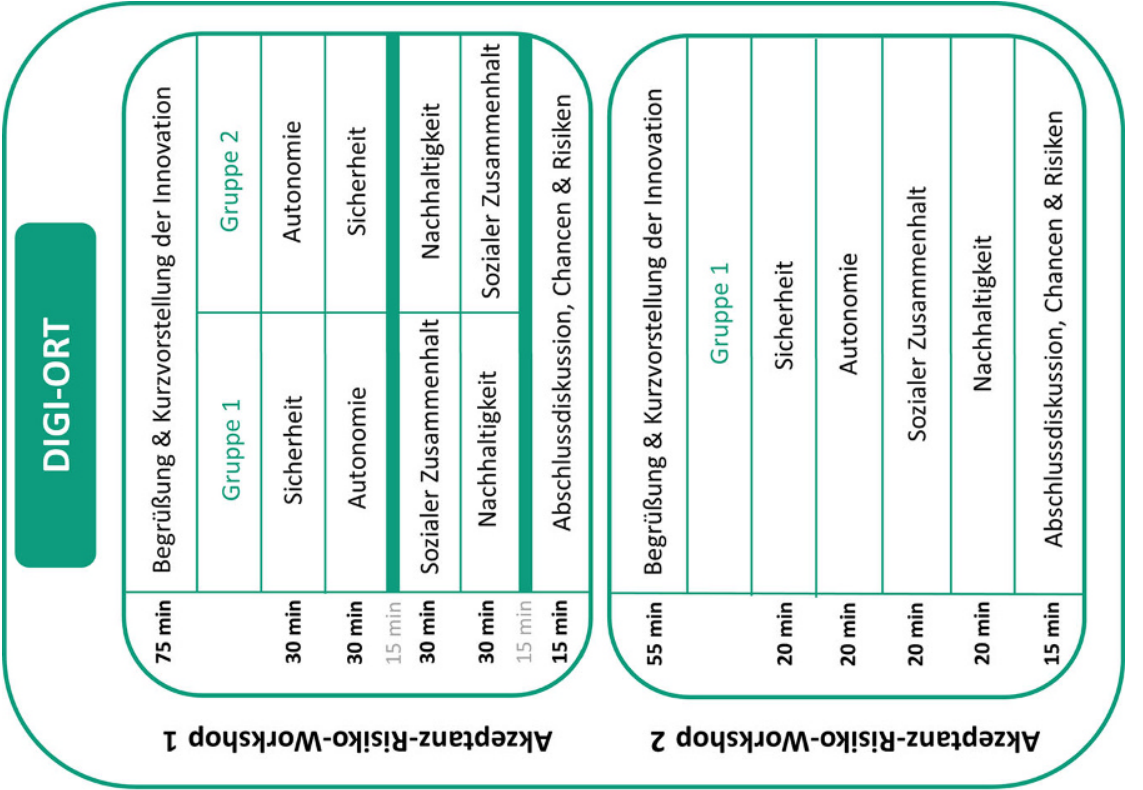

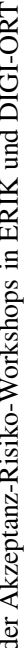

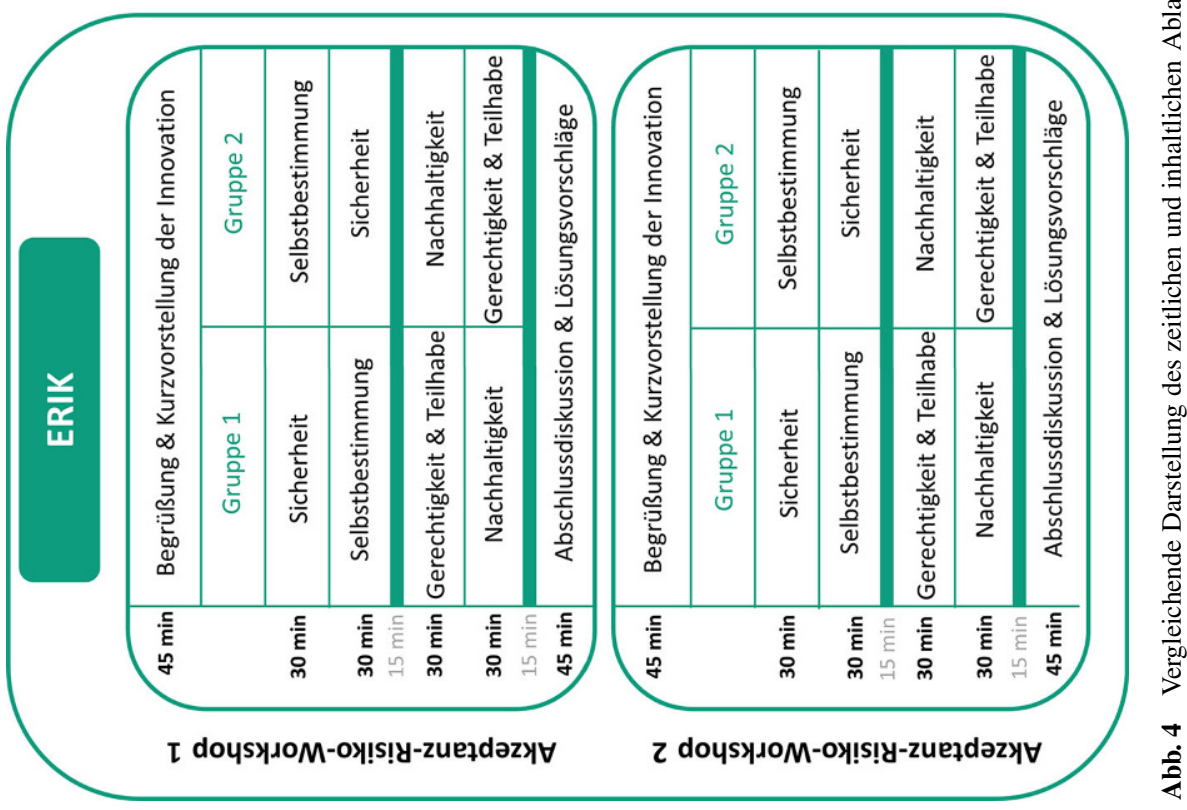


8 Teilnehmern besteht. Können bei ausreichender Teilnehmeranzahl zwei Gruppen gebildet werden, hat sich in der praktischen Umsetzung gezeigt, dass insbesondere gemischte Gruppen mit verschiedenen Nutzervertretern vorteilhaft sind, da in der Diskussion verschiedene Sichtweisen beleuchtet und - bei Anwesenheit von Projektbeteiligten - auch Rückfragen zum Entwicklungsprojekt direkt mit einbezogen werden können. Gerade in gemischten Gruppen, in denen die Vertreter der ,Vulnerable Consumers" (Baker et al. 2005) ihre Sichtweise einbringen konnten, konnte so ein verbessertes Verständnis der Nutzersicht erzielt werden, indem Ängste, Sorgen und Befürchtungen dieser Gruppen gehört und für die weitere Projektentwicklung aufgenommen wurden. Dies wiederum kann nur dann erreicht werden, wenn die Workshop-Moderatoren über eine offene, wertschätzende, empathische und doch neutrale Grundhaltung verfügen, die auf Seiten der Workshop-Teilnehmer das nötige Vertrauen schafft, um Gedanken, Ängste und Sorgen im geschützten Raum des Workshops frei zu äußern.

Während Dauer und Ablauf der Akzeptanz-Risiko-Workshops flexibel gehandhabt werden können, sollte darauf geachtet werden, bei der Durchführung keine inhaltlichen Änderungen vorzunehmen. Für eine ganzheitliche Betrachtung sollten alle vier Themenbereiche diskutiert werden, um sowohl die Position des Individuums in der Gesellschaft als auch überindividuelle und gesamtgesellschaftliche Themen $\mathrm{zu}$ adressieren. Hier hat sich jedoch in der praktischen Anwendung im Rahmen der Akzeptanz-Risiko-Workshops in ERIK gezeigt, dass die ursprüngliche Benennung der Themenbereiche und ihrer Unterkategorien teilweise zu Verständnis- und Interpretationsschwierigkeiten seitens der Teilnehmer führte. Im Forschungsprojekt DIGI-ORT wurden die betroffenen Themenbereiche und Unterkategorien daher umbenannt (siehe Abb. 4).

Als zentrale Stärke des HEART-Ansatzes hat sich die Möglichkeit einer quantitativen Bewertung von sozio-ethischen Themenaspekten erwiesen, die ein potenzielles Akzeptanzrisiko einer geplanten Technik auf Seiten der Nutzer darstellen. Betrachtet man die in Tab. 1 dargestellten Handlungsfelder, die in ERIK als Aspekte mit hohem Akzeptanzrisiko identifiziert wurden, so handelt es sich vielfach um Aspekte, die bereits bekannt sind und ohnehin in jedem Technikentwicklungsprojekt berücksichtigt werden (z. B. Vermeidung von Datenzugriff durch Unbefugte). Man könnte nun also damit argumentieren, dass es sich um eine redundante Information handelt. Die Information an sich mag tatsächlich redundant sein, sie erhält dank des HEARTAnsatzes nun aber ein Gewicht - nämlich das Gewicht des Nutzers, der durch seine Bewertung zeigt, wie wichtig ihm ein bestimmter Aspekt ist. Durch den HEARTAnsatz kann also ein verbessertes, quantifizierbares Verständnis der Nutzersicht erreicht werden, das letztlich auch dazu dienen kann, in einer frühen Projektphase ggf. Anpassungen vorzunehmen, um die Nutzerakzeptanz und damit den Erfolg des Technikentwicklungsprojekts zu gewährleisten.

\subsection{Schwächen des HEART-Ansatzes}

Diese Stärke der quantitativen Bewertung von sozio-ethischen Akzeptanzrisiken kann auch gleichzeitig als Schwäche des HEART-Ansatzes gesehen werden: Für eine vollumfassende quantitative Bewertung der im Akzeptanz-Risiko-Workshop 
erarbeiteten Aspekte ist zwingend ein zweistufiges Verfahren, bestehend aus Workshop und nachgelagertem Bewertungsfragebogen nötig. Zwar wurde im Rahmen von ERIK versucht, die erarbeiteten Aspekte bereits während der Workshops mittels Bepunktung hinsichtlich ihrer Wichtigkeit zu kategorisieren, dies ließ sich jedoch aufgrund der Vielzahl der teilweise auch doppelt genannten Aspekte nicht erfolgreich realisieren. Der nachgelagerte Bewertungsfragebogen selbst erfordert wiederum das Vorliegen der Workshop-Auswertungen, wodurch für Durchführung und Auswertung der beiden Komponenten ein Zeitraum von ca. 6 Wochen (abhängig u.a. von der Menge des zu transkribierenden Tonmaterials) veranschlagt werden muss. Diese Zeitdauer ist zum einen im Hinblick darauf einzukalkulieren, dass in gegenwärtigen IT-Entwicklungsprojekten Entwicklungsinhalte und -ablauf häufig bereits weitgehend definiert sind, bevor eine Nutzerintegration z.B. im Rahmen des HEARTAnsatzes überhaupt erfolgt. Können nun diese Erkenntnisse aus der Nutzerintegration - bedingt durch benötigte Auswertungszeit - erst zeitversetzt ins Projekt zurückfließen, bedeutet dies, dass ggf. zentrale Punkte nicht mehr im Projektplan berücksichtigt werden können. Zum zweiten muss aufgrund der Auswertungszeit auch damit gerechnet werden, dass der Bewertungsfragebogen nicht mehr von allen Workshop-Teilnehmern beantwortet wird, was sich wiederum auf die Generalisierbarkeit der Ergebnisse und damit indirekt auch deren Verwendung auswirkt. Trotz dieser Einschränkungen hat sich der nachgelagerte Bewertungsfragebogen als geeignete Methode erwiesen, da er - im Vergleich zu Priorisierungsversuchen während des Akzeptanz-Risiko-Workshops - eine höhere Validität und Reliabilität aufweist: Zum einen fließen hier sämtliche erarbeiteten Aspekte in die Bewertung ein, zum anderen kann durch die Zusammenfassung von doppelt genannten oder inhaltlich ähnlichen Aspekten eine Verzerrung in der Bewertung vermieden werden. Nicht zuletzt stellt das Ergebnis in Form eines zahlenbasierten Ratings eine fundierte Priorisierungsgrundlage für die weiteren Arbeitsschritte innerhalb des Entwicklungsprojekts dar.

\subsection{Weiterentwicklung und Optimierung des HEART-Ansatzes}

Als zentrale Ansatzpunkte für eine Weiterentwicklung und Optimierung des HEART-Ansatzes können daher vor allem folgende zwei Aspekte gesehen werden: Zum einen sollte der Auswertungsprozess des Akzeptanz-Risiko-Workshops beschleunigt werden, um einem möglichen Dropout der Teilnehmer entgegenzuwirken. Zum anderen sollte versucht werden, bereits für den Akzeptanz-RisikoWorkshop eine größere Teilnehmerzahl zu rekrutieren, sodass eine größere Ausgangsstichprobe für den nachgelagerten Bewertungsfragebogen zur Verfügung steht. Eine Alternative könnte auch darin bestehen, die Stichprobe für den Bewertungsfragebogen auf Teilnehmer außerhalb des Akzeptanz-Risiko-Workshops auszudehnen und so „die breite Masse“ einzubeziehen. Zur Sicherstellung des Verständnisses müsste dem Bewertungsfragebogen hier sowohl eine kurze Beschreibung des geplanten Entwicklungsvorhabens als auch der vier sozio-ethischen Themenbereiche, die den Gliederungsrahmen des Fragebogens bilden, vorangestellt werden.

Weiterer Forschungsbedarf besteht ferner hinsichtlich der Frage, wie die Ergebnisse aus dem HEART-Ansatz am besten Eingang in das Entwicklungsprojekt finden 
können und wie eine Übersetzung der bisherigen tabellarischen Dokumentation der Ergebnisse in den Entwicklerkontext erfolgen kann.

\subsection{Mehrwert des HEART-Ansatzes}

Wie einleitend bereits angeführt, kann der HEART-Ansatz ergänzend zu Scrum bzw. Design Thinking-Methoden eingesetzt werden. Eine Abgrenzung erfolgt dabei durch die unterschiedlichen Schwerpunkte der Methoden: Während bei Scrum eher der Entwicklungsprozess aus IT-Sicht im Vordergrund steht und jegliche Arten von Anforderungen der Stakeholder über die Person des Product Owners kanalisiert werden, fokussiert Design Thinking zwar auf die Einbindung der Nutzer, setzt aber häufig bereits in der Phase der Konzeptentwicklung an. Der HEART-Ansatz dagegen kommt erst zum Einsatz, wenn die Phase der Konzeptentwicklung bereits abgeschlossen ist und die technische Entwicklung und Umsetzung z. B. im Rahmen von Scrum beginnt. HEART beleuchtet in dieser Phase gemeinsam mit den Nutzervertretern das abgesteckte Feld ethisch, sozial und rechtlich kritischer Aspekte, das in Scrum oder Design Thinking-Methoden i.d.R. nicht explizit adressiert wird, und geht dabei durch die skalierbare Bewertung dieser Aspekte durch die Nutzervertreter über eine reine Erhebung hinaus. Darüber hinaus liegt der Mehrwert des HEARTAnsatzes in folgenden drei Aspekten:

1. Der Mensch steht im Mittelpunkt: Gemäß der sozialkonstruktivistischen Theorie der Technik (Berger und Luckmann 2005) werden der Erkenntnisgegenstand und die damit verbundene Wahrnehmung einer Technik durch den Betrachter erzeugt. Um eine Technik somit ganzheitlich einschätzen zu können, muss das zugehörige sozio-technische System mit seinen potenziellen Nutzergruppen verstanden werden. HEART gewährleistet dies durch die Integration unterschiedlichster Stakeholder: sowohl primäre und sekundäre Nutzer als auch Forscher, Industriepartner und politische Entscheidungsträger.

2. Besondere Berücksichtigung sogenannter ,,Vulnerable Consumers “: Durch explizite Berücksichtigung unterschiedlicher Perspektiven verschiedener Anspruchsgruppen ist HEART auch für schutzbedürftige Personen anwendbar ist: Risikofaktoren können etwa Alter, Unerfahrenheit, Lern- oder körperliche Behinderungen, niedriges Einkommen oder Lesefähigkeit, kulturelle Barrieren, psychische Erkrankungen, Beziehungsausfälle, Leben in Gebieten ohne Internet oder Gesundheitsprobleme sein (Baker et al. 2005).

3. Möglichkeit zum Wachstumsdenken „Growth-Mindset“ (Dweck 2016): HEART eröffnet die Möglichkeit, dass im Rahmen des Entwicklungsprozesses Dinge besser verstanden werden und ,Fehler“ als Möglichkeit gesehen werden, etwas Neues auszuprobieren und sich weiterzuentwickeln. Mit einem Growth Mindset können negative Emotionen, die etwa durch unerwartete Probleme oder Kritik verursacht werden, ausgeglichen werden (Myers et al. 2016). 


\section{Fazit}

Es zeigte sich nach Durchführung und Auswertung von vier Workshops mit insg. 41 Teilnehmern, dass - unter Einbezug einer breiten Basis an Zielgruppen (etwa Entwickler, interdisziplinäre Projektpartner, Betroffene) - der traditionelle ,technology push"-Ansatz im Bereich der Technikentwicklung durch einen bottom-upAnsatz (,demand pull“) bereichert werden kann. Das entwickelte HEART-Konzept erweist sich im Kontakt mit nicht-wissenschaftlichen, nicht-technikversierten oder nicht-projekterfahrenen Personen als geeignet, deren Sichtweisen auf Technik und ihre sozio-ethischen Risikofaktoren zu erheben und auf diese Weise integrative Forschung in Technikentwicklungsprozessen umzusetzen. Sein Einsatz kann sowohl zu Beginn technischer Entwicklungsprojekte als auch in Stadien, in denen noch keine Projektidee vorhanden ist, zur Ermittlung des Bedarfs und der Relevanz eingesetzt werden. Sozio-ethische Betrachtungen von Akzeptanzrisiken in Technikentwicklungsprozessen? - Mehr als eine Zeitverschwendung!

Funding Open Access funding provided by Projekt DEAL.

Open Access Dieser Artikel wird unter der Creative Commons Namensnennung 4.0 International Lizenz veröffentlicht, welche die Nutzung, Vervielfältigung, Bearbeitung, Verbreitung und Wiedergabe in jeglichem Medium und Format erlaubt, sofern Sie den/die ursprünglichen Autor(en) und die Quelle ordnungsgemäß nennen, einen Link zur Creative Commons Lizenz beifügen und angeben, ob Änderungen vorgenommen wurden.

Die in diesem Artikel enthaltenen Bilder und sonstiges Drittmaterial unterliegen ebenfalls der genannten Creative Commons Lizenz, sofern sich aus der Abbildungslegende nichts anderes ergibt. Sofern das betreffende Material nicht unter der genannten Creative Commons Lizenz steht und die betreffende Handlung nicht nach gesetzlichen Vorschriften erlaubt ist, ist für die oben aufgeführten Weiterverwendungen des Materials die Einwilligung des jeweiligen Rechteinhabers einzuholen.

Weitere Details zur Lizenz entnehmen Sie bitte der Lizenzinformation auf http://creativecommons.org/ licenses/by/4.0/deed.de.

\section{Literatur}

Baker SM, Gentry GW, Rittenburg TL (2016) Building understanding of the domain of consumer vulnerability. J Macromark 25:128-139

Berger PL, Luckmann T (2005) Die gesellschaftliche Konstruktion der Wirklichkeit

Dweck C (2016) What having a "growth mindset" actually means. Harv Bus Rev 13:213-226

Kollmann T (1998) Akzeptanz innovativer Nutzungsgüter und -systeme: Konsequenzen für die Einführung von Telekommunikations- und Multimediasystemen. Springer, Wiesbaden

Königstorfer J (2008) Akzeptanz von technologischen Innovationen; Nutzungsentscheidungen von Konsumenten dargestellt am Beispiel von mobilen Internetdiensten. Gabler, Wiesbaden

Kranzberg M (1986) Technology and History: "Kranzberg's Laws". Technol Cult 27:544-560

Lucke D (1995) Akzeptanz: Legitimität in der „Abstimmungsgesellschaft“. VS, Wiesbaden

Manzeschke A (2015) MEESTAR: ein Modell angewandter Ethik im Bereich assistiver Technologien. Technisierung des Alters - Beitrag zu einem guten Leben, S 263-283

Mayring P (2002) Einführung in die qualitative Sozialforschung; Eine Anleitung zu qualitativem Denken. Beltz, Weinheim

Myers CA, Wang C, Black JM, Bugescu N, Hoeft F (2016) The matter of motivation: Striatal resting-state connectivity is dissociable between grit and growth mindset. Soc Cogn Affect Neurosci $11: 1521-1527$ 
Palm K, Hansson J (2018) Influencing project work: exploring the potentials of participatory research. J Work-Appl Manag 10:63-73

Simon B (2001) E-Learning an Hochschulen: Gestaltungsräume und Erfolgsfaktoren von Wissensmedien. Eul Verlag, Lohmar, Köln, Durbusch

Simon M, Strehler M, Kirst S, Schmitt-Rüth S, Lang N (2019) Akzeptanzrisiken in der Mensch-RoboterInteraktion - Nutzerzentrierte Erhebung von ethischen, rechtlichen und sozialen Implikationen (ELSI) am Beispiel einer Roboterplattform für Kinder mit eingeschränkten sozio-emotionalen Fähigkeiten. Mensch und Computer 2019 - Workshopband. Gesellschaft für Informatik e. V., Bonn

Stahl BC (2011) IT for a better future; How to integrate ethics, politics and innovation. J Inf Commun Ethics Soc 9:140-156

Weber K (2016) MEESTAR2. Ein erweitertes Modell zur ethischen Evaluierung soziotechnischer Arrangements. Technische Unterstützungssysteme, die die Menschen wirklich wollen. Helmut-SchmidtUniversität, Hamburg, S 317-326 